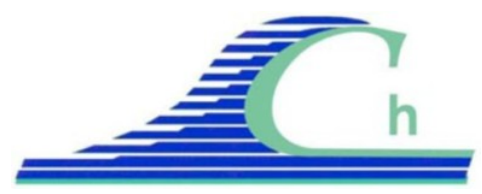

XII ${ }^{\text {ìmes }}$ Journées Nationales Génie Côtier - Génie Civil

Cherbourg, 12-14 juin 2012

DOI:10.5150/jngcgc.2012.045-S @ Editions Paralia CFL

disponible en ligne - http://www.paralia.fr - available online

\title{
Vulnérabilité et impacts des risques associés à l'élévation du niveau de la mer dans la plaine littorale de Saidia - Cap de l'Eau (Maroc nord-est)
}

\author{
Abdelkader SBAI ${ }^{1}$, Hicham LASGAA ${ }^{1}$ \\ 1. Université Mohamed I ${ }^{\mathrm{er}}$, Laboratoire de Géomatique, Patrimoine et Développement, \\ 60000, Oujda, Maroc. \\ Sbai_abdelkader@yahoo.fr
}

\section{Résumé :}

La plaine littorale de Saidia - Cap de l'Eau est l'une des régions de la côte méditerranéenne du Maroc les plus vulnérables à une montée probable du niveau de la mer à cause de ses caractéristiques physiques. L'analyse des zones d'inondation potentielle et les estimations de la vulnérabilité sont basées sur une approche empirique à partir des évaluations faites par l'IPCC sur l'élévation du niveau de la mer et les scénarios extrêmes de la marée astronomique. Les résultats indiquent que pour une montée de $1 \mathrm{~m}$ du niveau de la mer (scénario optimiste), une partie de la plaine littorale (11\%) serait submergée. Dans un scénario de remontée du niveau de la mer critique (3 m), ce chiffre monte à 56\%. L'analyse de la côte entière indique que plus de $90 \%$ des constructions urbaines situées en dessous d'une altitude de $3 \mathrm{~m}$ seraient affectées sévèrement par les changements dans le niveau de la mer. Actuellement, un tiers de la côte est considéré comme région à haute vulnérabilité. Vu les pertes sévères prédites par les scénarios simulés, les stratégies de la réponse qui identifient les options de l'adaptation les plus appropriées doivent être développées.

Mots-clés : Risque d'inondation côtière - montée du niveau de la mer - Maroc nord-est

\section{Introduction}

Le dernier rapport du Groupe Intergouvernemental sur le Changement Climatique conclut que le climat de la planète connait indubitablement un réchauffement rapide dû partiellement aux activités humaines (IPCC, 2007). D'après ce rapport, les scénarios de l'élévation du niveau de la mer sont de grande inquiétude. L'accélération du taux de l'élévation du niveau de la mer rend non seulement la possibilité d'impacts plus intenses (érosion côtière, pertes d'habitat, intrusion saline), mais pourrait aussi provoquer une disparition complète des plages sableuses et des marécages.

Un tel impact dépend des particularités locales et se produit différemment. De plus, l'adaptation des régions côtières représente un plus grand défi aux pays en voie de développement, à cause des ressources économiques limitées.

$\mathrm{Au} \mathrm{Maroc,} \mathrm{les} \mathrm{tendances} \mathrm{de} \mathrm{l'élévation} \mathrm{du} \mathrm{niveau} \mathrm{de} \mathrm{la} \mathrm{mer} \mathrm{varient} \mathrm{d'un} \mathrm{secteur} \mathrm{à}$ l'autre selon le contexte géologique (zone en subsidence, en soulèvement...). 
WARRICK et al. (1996) avancent des valeurs à caractère global de 4,9 à 8,6 mm/an. L'objectif de cette étude est d'évaluer la vulnérabilité de la plaine littorale de Saidia Cap de l'Eau face à la submersion simulée à partir des scénarios possibles de l'IPCC (2001 ; 2007). Le scénario de $7 \mathrm{~m}$ avancé par SNOUSSI et al. (2008) est fortement exagéré. Après avoir identifié les enjeux liés à l'élévation du niveau de la mer, nous avons dressé une cartographie prévisionnelle des risques de submersion et analysé les impacts potentiels de ce phénomène.

\section{Description de la zone d'étude}

La plaine littorale de Saidia - Cap de l’Eau est localisée sur le littoral méditerranéen oriental du Maroc (figure 1) et s'étend sur le delta de l'Oued Moulouya. Elle combine des topographies basses (0 à $6 \mathrm{~m}$ ), une urbanisation intense et un intérêt écologique, touristique et économique important. En outre, elle présente plusieurs conflits d'usage des ressources côtières et est une des premières régions au Maroc pour faire l'objet d'études intégrées (SBAI \& MELHAOUI, 2009).

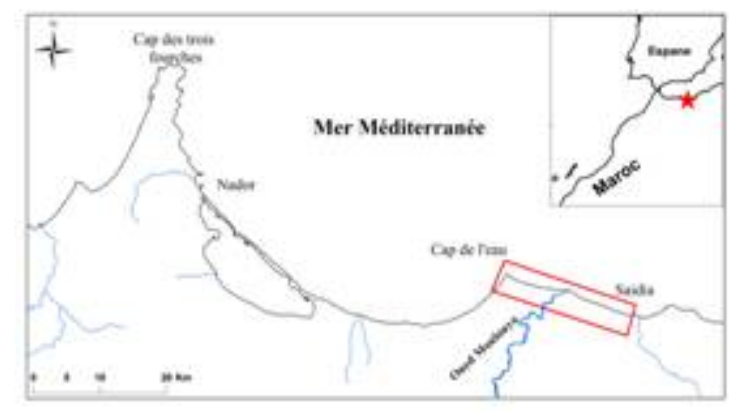

Figure 1. Localisation de la zone d'étude sur le littoral méditerranéen du Maroc.

La région présente un climat méditerranéen sec et chaud en été et humide et frais en hiver avec des écarts thermiques saisonniers importants et des précipitations peu abondantes (319 mm à Saïdia). Les températures moyennes annuelles sont de l'ordre de $17-18^{\circ} \mathrm{C}$. La vitesse moyenne du vent est de $4,3 \mathrm{~m} / \mathrm{s}$ à Nador-Taouima.

Les marées enregistrées dans la région sont toutes semi-diurnes et classées comme microtidales. La hauteur des vagues varie selon les sites, avec des valeurs de 0.3 à 2,5 pour Melilia (http://www.puertos.es).

Les principaux écosystèmes sont les cordons dunaires récents et anciens (figure 2), la forêt, et le Site d'Intérêt Biologique et Ecologique (SIBE) de la Moulouya. Le système hydrographique est bien drainé, avec deux principaux oueds (la Moulouya et Kiss).

La plaine littorale de Saidia - Cap de l'Eau connaît une érosion importante : $8 \mathrm{~mm} / \mathrm{an}$ à l'embouchure de la Moulouya et 0.5 m/an à Saidia (SBAI \& BOUAZZA, 2002). L'anthropisation de la côte a des impacts géomorphologiques et environnementaux catastrophiques. 


\section{XII ${ }^{\text {èmes }}$ Journées Nationales Génie Côtier - Génie Civil \\ Cherbourg, 12-14 juin 2012}

La présence au niveau de la plaine côtière d'une nappe phréatique salée et de sols argileux salés, rend difficile sa mise en valeur agricole. En revanche, la plage de sable présente un grand intérêt pour le tourisme balnéaire. La ville de Saïdia est depuis longtemps un lieu de tourisme national. Depuis 2001, son statut a été renforcé par l'adoption par le Gouvernement marocain du plan Azur qui avait pour objectif de tripler le nombre de touristes accueillis chaque année pour le porter à dix millions en 2010. Ce plan prévoyait, à l'échelle du Maroc, la création de six stations touristiques : Saïdia, Mogador, Lixus, Mazagan, Taghazout et Plage-Blanche.

La Nouvelle Station Touristique de Saïdia (NSTS), intitulée Mediterrania-Saïdia s'étend sur une superficie de 713 ha. C'est le plus grand chantier touristique jamais réalisé au Maroc. Il comporte une marina, des hôtels de hauts standings, des villas, des appartements, des résidences touristiques, des villages touristiques, trois parcours de golf avec 18 trous chacun, un aquapark, un centre de thalassothérapie, un palais des congrès, une clinique et des équipements sportifs.

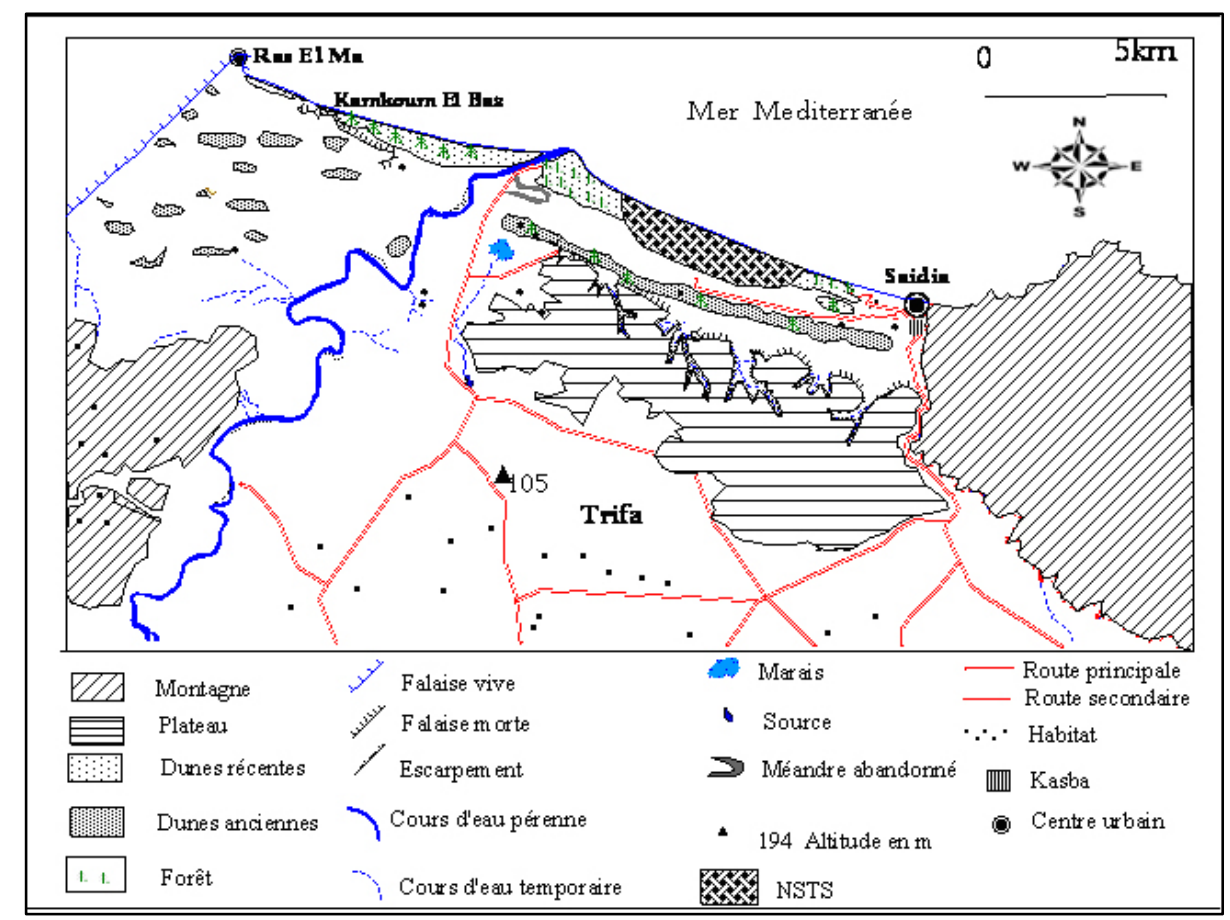

Figure 2. Principales unités géomorphologiques de la zone d'étude.

\section{Méthodologie}

\subsection{Evaluation des différents niveaux d'inondation}

L'approche empirique utilisée pour déterminer les niveaux d'inondation dans les régions côtière et d'estuaire est basée sur la formule de HOOZEMANS et al. (1993).

$\mathrm{Dft}: \mathrm{MHW}+\mathrm{S}_{\mathrm{t}}+\mathrm{W}_{\mathrm{f}}+\mathrm{P}_{\mathrm{f}}$ 
Avec:

MHW : Niveau moyen des hautes eaux ;

$\mathrm{S}_{\mathrm{t}}$ : Elévation relative du niveau marin ;

$\mathrm{W}_{\mathrm{f}}$ : Hauteur des houles de tempêtes responsables des inondations ;

$\mathrm{P}_{\mathrm{f}}$ : Elévation du niveau marin sous l'effet d'une baisse de pression.

Les étapes méthodologiques proposées par HOOZEMANS et al. (1993) et appliquées par SNOUSSI et al. (2008), ont été suivies, pour déterminer les niveaux d'inondation par la somme des facteurs impliqués dans l'élévation du niveau de la mer. Par conséquent, les contributions des phénomènes induits par le niveau d'eau extrême ont été simulées: haute ligne d'eau maximale au cours des 20 dernières années; montée du niveau de la mer due aux tempêtes : surcôtes et haute vague (http://www.puertos.es); montée du niveau de la mer projeté pour le prochain siècle (IPCC, 2007) qui peut être optimiste ( $+0.5 \mathrm{~m}$ pour 2100, approximativement $5 \mathrm{~mm} / \mathrm{an})$ ou critique ( $+1.0 \mathrm{~m}$ pour 2100, approximativement $10 \mathrm{~mm} / \mathrm{an})$. Ces scénarios ont été adaptés des scénarios de l'IPCC (B1 et A2), vu que la montée du niveau de la mer pour le prochain siècle peut être jusqu'à approximativement deux fois la projection maximale trouvée dans ce rapport (ROHLING et al., 2008).

La méthode utilisée ne tient pas compte de la subsidence et des mouvements tectoniques, alors que le littoral étudié appartient à une marge active. Cette activité tectonique se poursuit encore de nos jours comme cela est attesté par les nombreux séismes qui ont affecté la région. Malheureusement, nous ne disposons pas de données sur les taux de subsidence et sur la cinématique de la région. Les estimations restent donc limitées.

\subsection{Topographie et utilisation des sols}

Les données de l'élévation ont été extraites de la restitution aérienne de 2006. Un modèle numérique d'élévation (DEM) a été produit de l'interpolation des données de l'élévation par réseau triangulaire irrégulier (TIN), et une base de données de points de contrôle au sol a été mesurée par un système de positionnement global (GPS).

La résolution horizontale du DEM est de quelques centimètres, ce qui permet de représenter correctement des traits étroits tels que les routes, les dunes, les bâtiments... ; l'exactitude verticale est de $0.4 \mathrm{~m}$. Ces données ont été intégrées dans un SIG (ArcGIS 9.1) pour classer et dresser une carte de la typologie des terres menacées par les inondations potentielles. Les images satellitales et les photographies aériennes ont été rectifiées et traitées pour numériser les lignes du niveau d'inondation.

\section{Résultats}

\section{1 Établissement des zones d'inondation potentielles}




\section{XII ${ }^{\text {èmes }}$ Journées Nationales Génie Côtier - Génie Civil \\ Cherbourg, 12-14 juin 2012}

Le SIBE de la Moulouya est le site avec l'altitude la plus basse dans la zone d'étude, $(0-1 \mathrm{~m})$. Dans ce secteur, on peut observer que partout la côte entière est sujette à inondation, sauf une portion de la dune légèrement sauvegardée. Néanmoins, il y a encore une perte de plage sableuse estimée à $8 \mathrm{~m} / \mathrm{an}$ par l'interprétation des photos aériennes à des dates différentes (SBAI \& BOUAZZA, 2002). Les changements les plus importants des différents scénarios apparaissent dans les régions qui entourent la Moulouya et les marais adjacents. Plusieurs simulations réalisées à partir d'un modèle numérique d'élévation montrent que l'élévation du niveau marin pourrait entrainer la submersion de l'ensemble du SIBE d'ici la fin du siècle. Une des conséquences pourrait être la substitution du marais salé au marais doux et donc une perte de la biodiversité végétale.

Saidia Mediterranéa présente la côte la plus étendue dans la zone d'étude. C'est un site avec des altitudes faibles surtout après la démolition de la dune. En conséquence, dans un scénario d'une montée du niveau de la mer de $2 \mathrm{~m}$, il est estimé que la région inondée occuperait approximativement 500 ha; pendant que ce serait 650 ha, dans le scénario critique avec une montée de $3 \mathrm{~m}$.

La ville de Saidia présente la plus grande région parmi les secteurs étudiés. La zone d'inondation potentielle est montrée comme polygones pour les scénarios optimiste et critique (figure 3, 4 et 5). Les changements les plus importants des deux scénarios apparaissent sur la bande côtière située à proximité de Saidia et à Caracas, où la démolition de la dune a rendu cette partie extrêmement vulnérable.

Le centre de Ras El Ma est localisé à plus de $15 \mathrm{~m}$ au-dessus du niveau de la mer. Cette zone ne se présente pas comme une région très sujette aux inondations. Cependant, la bande côtière en bas de la falaise présente un risque d'inondation : ancien centre de Ras El Ma et le port de pêche (figure 4).

\subsection{Analyse des zones à risque}

Les types d'utilisation du sol et les régions susceptibles d'être affectées par la submersion marine sont représentés dans les figures 3, 4 et 5 .

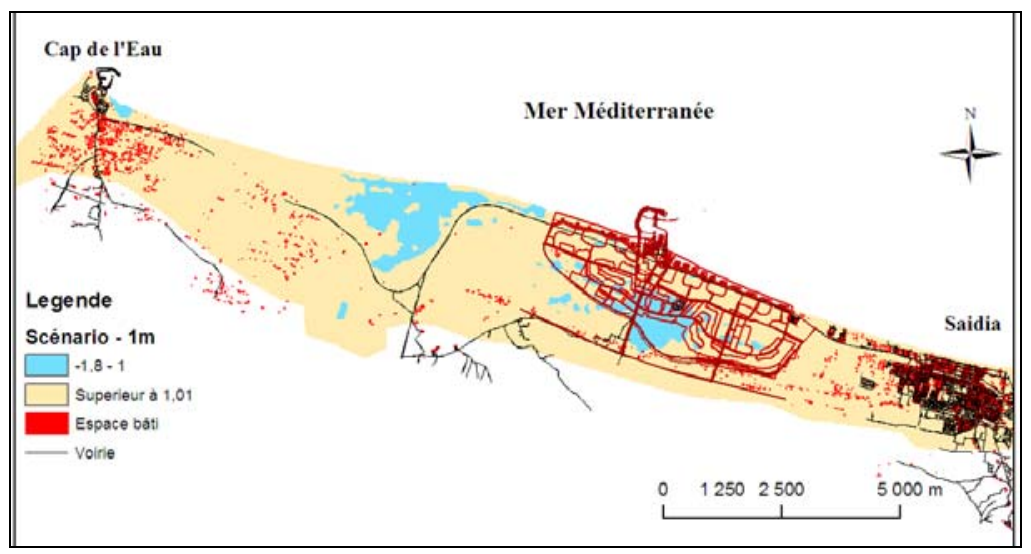


Figure 3. Zone à risque de submersion : scénario minimum de $1 \mathrm{~m}$.

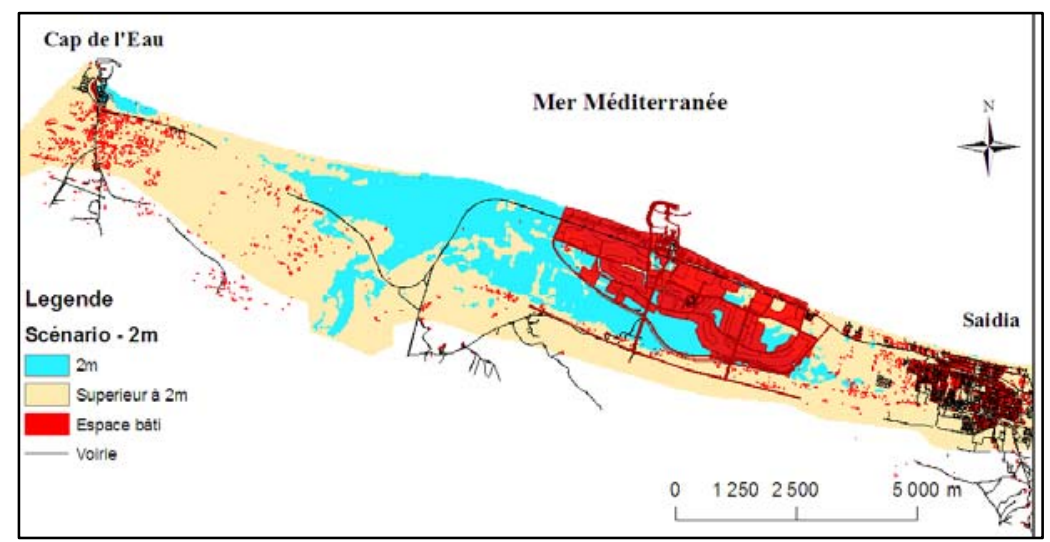

Figure 4. Zone à risque de submersion : scénario minimum de $2 \mathrm{~m}$.

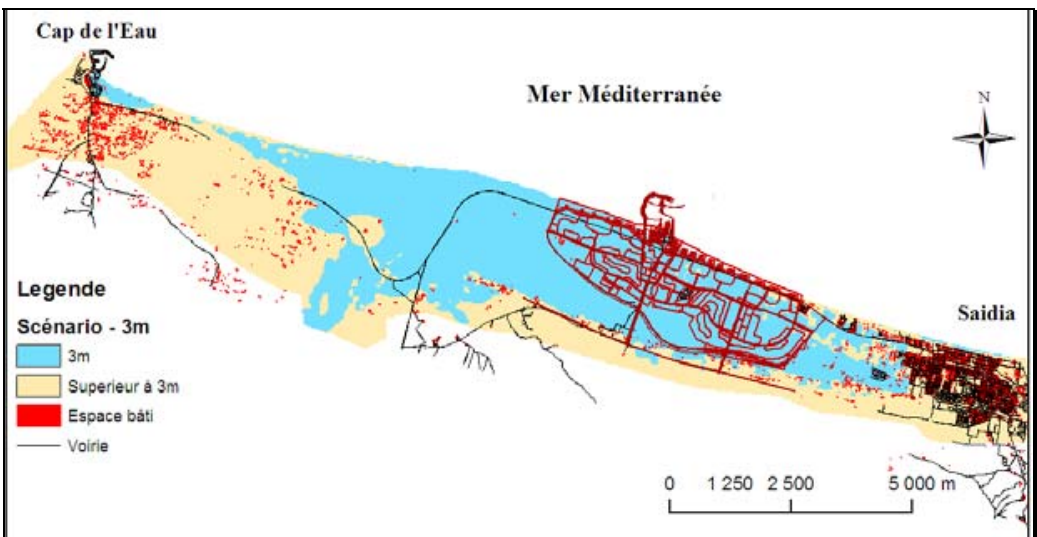

Figure 5. Zone à risque de submersion : scénario minimum de $3 \mathrm{~m}$.

Parmi les régions vulnérables du SIBE de la Moulouya, les marais attirent l'attention depuis qu'ils sont classés site de grande importance écologique.

Pour la station touristique Saidia Mediterranéa, dans un scénario optimiste, les régions relativement primitives - régions inoccupées et de plage - souffriraient du plus grand impact. En revanche, les régions avec bâtiments monteraient à 90\% des zones affectées dans un scénario critique.

À Saidia, la grande région avec bâtiments de moins de trois niveaux est très vulnérable à une augmentation du niveau de la mer. D'une perspective historique, nous mettons en valeur l'inondation potentielle de l'ancienne ville et de tout l'espace interdunaire. Ce patrimoine est vulnérable dans les deux scénarios.

Quant aux zones côtières mises en danger par les inondations à Ras El Ma, ce sont principalement les constructions basses qui se trouvent dans les zones à risque (Port de pêche et ancien centre). 


\section{XII ${ }^{\text {èmes }}$ Journées Nationales Génie Côtier - Génie Civil \\ Cherbourg, 12-14 juin 2012}

\section{Discussion}

L'analyse a été limitée à la plaine côtière qui est plus affectée par une élévation du niveau de la mer. Pour cette raison, il est clair que les chiffres et les discussions présentés sont sous-estimés et que les zones d'inondation potentielle dans la région étudiée sont plus grandes du fait du développement socio-économique futur et de l'évolution morphodynamique actuelle. Quant à l'impact sur l'environnement, nous signalons que des pertes écologiques importantes peuvent se produire.

Dans la perspective de l'élévation future du niveau marin, une grande partie des prairies arrière littorales subirait les conséquences d'une submersion par les eaux libres et/ou d'un engorgement par les eaux phréatiques à salinité accentuée. Dans ces conditions, ces végétations de prairies connaîtront une évolution régressive et le retour d'espèces caractéristiques du schorre.

Sur la côte de Saidia Mediterranéa (région pertinente d'un point de vue économique et touristique), la perte de plages et les dommages causés aux constructions par les courants littoraux sont communs. Il n'y a actuellement aucune construction de défense côtière placée le long du rivage. Comme mentionné précédemment, le rivage de la station touristique présente un haut degré de verticalisation qui augmente la magnitude de l'impact causée par l'inondation côtière.

Pour la ville de Saidia, parmi les régions sujettes à inondation dans les deux scénarios, l'attention est attirée à l'ancien centre-ville (Kasbah) qui représente un centre économique et administratif important de la ville avec une grande valeur historique et culturelle (origine de la ville de Saidia).

Bien que la région d'inondation potentielle soit étroite dans la bande côtière, l'intensification du processus érosif est notable, vu qu'il y a une rocade et des bâtiments localisés très près de la plage, ce qui expose les constructions basses aux processus morphodynamiques. Comme à la station touristique Saidia Mediterranéa, le rivage de la ville de Saidia présente un haut degré de verticalisation, à une telle ampleur que la perte de plage, ou le danger par érosion, impliquerait de grandes pertes économiques et une désorganisation sociale. Le trouble supplémentaire pourrait être impliqué par le fait que de nombreux habitants utilisent des fosses sceptiques qui pourraient devenir inutilisables à cause d'intrusion saline.

La mise en œuvre de politiques publiques pour la protection des zones côtières et d'inondation est suggérée dans deux optiques: (I) expansion et consolidation de connaissance scientifique du phénomène de submersion marine, et (II) gestion et établissement de mesures adaptatives pour minimiser son impact.

\section{Conclusion}

La plaine littorale de Saidia - Cap de l'Eau se présente comme une région très vulnérable à une élévation du niveau de la mer à cause de ses caractéristiques topographiques et socio-économiques. 
La submersion marine aura probablement des impacts sur la plaine littorale tant sur les plans économiques que environnementaux et morphologiques. Le plus important est la base de la connaissance et le meilleur est la prédiction des impacts qui résultent du changement climatique. Il faudra donc réaliser des plans de prévention des risques. Une fois les zones les plus vulnérables définies, les décideurs pourront ainsi établir les stratégies possibles et les priorités à mener pour minimiser les impacts potentiels de la montée du niveau de la mer et gérer au mieux le site à long terme.

\section{Références bibliographiques}

HOOZEMANS F.M.J., STIVE M.J.F., BIJLSMA L. (1993). A global vulnerability assessment: vulnerability of coastal areas to sea-level rise. 8th Symposium on Coastal and Ocean Management - Coastal Zone'93, 8, New Orleans, pp 390-404.

IPCC -Intergovernmental Panel on Climate Change. Climate Change- (2001). Climate Change 2001: The Scientific basis. In: Houghton, J.T., Ding, Y., Griggs, D.J., Noguer, M., van der Linden, P.J., Dai, X., Maskell, K., Johnson, C.A. (Eds.), Contribution of Working Group I to the Third Assessment Report of the Intergovernmental Panel on Climate Change. Cambridge University Press, Cambridge, United Kingdom and New York, NY, USA. 881 p.

IPCC -Intergovernmental Panel on Climate Change. Climate Change- (2007). The Physical Science Basis. Accessible at: http://www.ipcc.un.org.

ROHLING E.J., GRANT K., HEMLEBEN C., SIDDALL M., HOOGAKKER B.A.A., BOLSHAW M., KUCERA M. (2008). High rates of sea-level rise during the last interglacial period. Nature Geoscience, Vol. 1, pp 38-42. doi:10.1038/ngeo.2007.28

SBAI A., BOUAZZA A. (2002). Evolution géomorphologique et dynamique récente de la plaine littorale de Saïdia (Maroc Nord-est). In : Le littoral méditerranéen oriental : Diagnostic et choix de développement et d'aménagement. Colloque national, 13-14 février 2002, Oujda, pp 3-30.

SBAI A., MELHAOUI M. (2009). Etude socioéconomique de la zone côtière Saïdia Ras El Ma (MAROC) pour une gestion intégrée. Atelier international sur la gestion intégrée des zones côtières. Izmir-TURKEY, 20-22 October 2009.

SNOUSSI M., OUCHANI T., NIAZI S. (2008). Vulnerability assessment of the impact of sea- level rise and flooding on the Moroccan coast: The case of the Mediterranean eastern zone. Estuarine, Coastal and Shelf Science, Vol. 77, pp 206-213. doi:10.1016/j.ecss.2007.09.024

WARRICK R.A, LE PROVOST C, MEIER M.F, OERLEMANS J, WOODWORTH P.L (1996). Changes in sea level. In HOUGHTON J.T et al. (Eds). Climate Change 1995. The Science of Climate Change, Cambridge University Press, Cambridge (1996), pp 365-406. 